\title{
Molecular engineering and cosensitization for developing efficient solar cells based on porphyrin dyes with an extended $\pi$ framework
}

Dye-sensitized solar cells (DSSCs) have a promising future because of their low production cost, easy fabrication and relatively high solar energy conversion efficiency. In the past two decades, increasing interests have been focused on the improvement of cell efficiencies. Thus, many efficient sensitizers with donor- $\pi$-acceptor frameworks have been successfully developed. In this respect, porphyrins have been widely investigated because of their strong Soret bands $(400-450 \mathrm{~nm})$ and moderate $\mathrm{Q}$ bands $(550-650 \mathrm{~nm})$. The most prevalent obstacle encountered in designing efficient dyes is their weak absorption in the near-infrared region. To overcome this problem in typical porphyrin based dyes, the introduction of additional ethynylene bridges is a rational approach that extends the absorption bands to a longer wavelength region. However, one adverse effect of this approach is the aggravation of dye aggregation, which may lower the cell efficiencies. Thus, in most cases, the sensitizers with additional ethynylene bridges show decreased cell efficiencies.

To address this problem, a new design strategy has been recently reported in Angew Chem Int Ed by Yongshu Xie and He Tian et al. [1] in East China University of Science and Technology. In their studies, they developed four efficient porphyrin sensitizers, and molecular design strategies were employed to stepwise optimize the dye structures (Figure 1). Initially, four long alkoxyl chains were introduced to the ortho-positions of the meso-phenyl groups to suppress the dye aggregation. Then, an ethynylene bridge was introduced between the donor and the porphyrin framework to extend the absorption to longer wavelengths. Next, additional alkoxyl chains were introduced to the phenylene group attached to the donor group to further suppress the dye aggregation effect. This molecular design strategy proved to be effective for suppressing the dye aggregation caused by the additional ethynylene bridge. Finally, a non-porphyrin dye with a high $V_{\text {oc }}$ and strong absorption around $500 \mathrm{~nm}$ was used as the cosensitizer. Thus, significantly enhanced $J_{\text {sc }}$ and slightly enhanced $V_{\text {oc }}$ values were achieved with the highest cell efficiency of $10.45 \%$. This

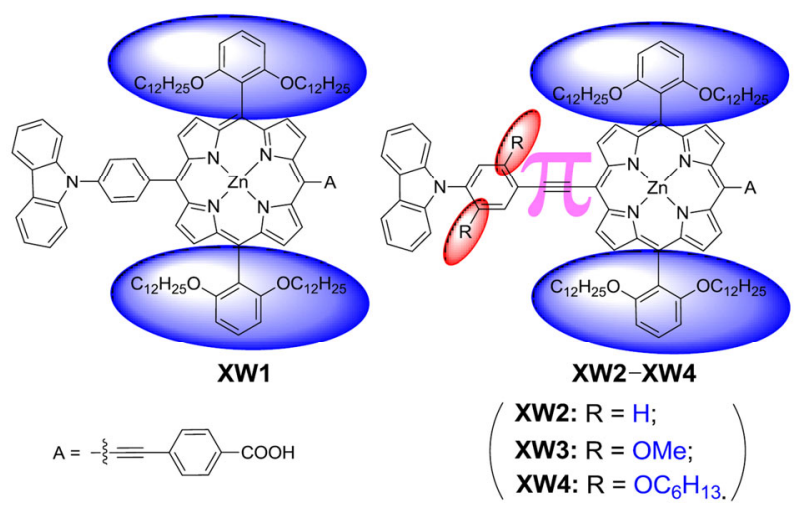

Figure 1 Molecular structures of XW1-XW4.

work presents an effective combination strategy of molecular design and cosensitization for developing efficient DSSCs. On the other hand, the best push-pull porphyrin sensitizers in DSSCs (PCE > 10\%) were typically composed of a diphenylamine based donor. In this work, the authors employed a carbazole electron donor, to obtain the highest cell efficiency of $10.45 \%$ that is significantly higher than the best efficiency of $5.74 \%$, which has been previously reported for porphyrin dyes containing a carbazole donor. Thus, this work demonstrates the opportunities of developing effective porphyrin sensitizers by employing electron donors other than diphenlyamine, and finds applications in the further development of new porphyrin based dyes with better performance.

LI Zhen \& LI QianQian Department of Chemistry, Wuhan University

1 Wang YQ, Chen B, Wu WJ, Li X, Zhu WH, Tian H, Xie YS. Efficient solar cells sensitized by porphyrins with extended conjugation framework and a carbazole donor: from molecular design to cosensitization. Angew Chem Int Ed, 2014, 53: 10779-10783 